\title{
Release of autoinhibition of ASEF by APC leads to CDC42 activation and tumor suppression
}

\author{
Natalia Mitin ${ }^{1,2}$, Laurie Betts ${ }^{2}$, Marielle E Yohe ${ }^{2}$, Channing J Der ${ }^{1,2}$, John Sondek ${ }^{1,2,3}$, and \\ Kent L Rossman ${ }^{1,2}$ \\ ${ }^{1}$ Lineberger Comprehensive Cancer Center, University of North Carolina School of Medicine, \\ Chapel Hill, North Carolina 27599, USA. \\ 2Department of Pharmacology, University of North Carolina School of Medicine, Chapel Hill, North \\ Carolina 27599, USA. \\ ${ }^{3}$ Department of Biochemistry and Biophysics, University of North Carolina School of Medicine, \\ Chapel Hill, North Carolina 27599, USA.
}

\begin{abstract}
Autoinhibition of the Rho guanine nucleotide exchange factor ASEF is relieved by interaction with the APC tumor suppressor. Here we show that binding of the armadillo repeats of APC to a "core APC-binding' (CAB) motif within ASEF, or truncation of the SH3 domain of ASEF, relieves autoinhibition, allowing the specific activation of CDC42. Structural determination of autoinhibited ASEF reveals that the SH3 domain forms an extensive interface with the catalytic DH and $\mathrm{PH}$ domains to obstruct binding and activation of $\mathrm{CDC} 42$, and the $\mathrm{CAB}$ motif is positioned adjacent to the SH3 domain to facilitate activation by APC. In colorectal cancer cell lines, full-length, but not truncated, APC activates CDC42 in an ASEF-dependent manner to suppress anchorage-independent growth. We therefore propose a model in which ASEF acts as a tumor suppressor when activated by APC and inactivation of ASEF by mutation or APC truncation promotes tumorigenesis.
\end{abstract}

The adenomatous polyposis coli (APC) protein is a negative regulator of the Wnt signaling pathway and promotes the phosphorylation and degradation of $\beta$-catenin ${ }^{1}$. The majority of colorectal cancers (CRCs) harbor C-terminally truncated forms of APC that retain the Nterminal coiled-coil domain and the highly conserved armadillo repeat region $\left(\mathrm{APC}^{\mathrm{Arm}}\right)^{2}$ and are unable to regulate the degradation of $\beta$-catenin ${ }^{3}$. Recent studies suggest that APC regulates cytoskeletal dynamics to influence cellular migration, cell division, polarization and adhesion, and it seems increasingly likely that deregulated cytoskeletal dynamics, together with enhancement of transcription by $\beta$-catenin, potentiate tumor formation and progression upon APC truncation.

\section{(c) 2007 Nature Publishing Group}

Correspondence should be addressed to C.J.D. (E-mail: cjder@med.unc.edu) and K.L.R. (E-mail: krossman@med.unc.edu). AUTHOR CONTRIBUTIONS

N.M. and K.L.R. conceived, analyzed and performed experiments and wrote the manuscript. L.B. assisted with the structural determination of ASEF. M.E.Y. contributed ideas and assisted with construct design and protein purification. J.S. and C.J.D. contributed ideas and evaluated and discussed data.

Accession codes. Protein Data Bank: Coordinates and structure factors have been deposited with accession code 2PZ1. 
APC may regulate cytoskeletal networks by binding to microtubules and to proteins implicated in reorganization of the actin cytoskeleton, such as the Rho effectors mDia and IQGAP1 and the Rho guanine nucleotide exchange factor (RhoGEF) 'APC-stimulated guanine nucleotide exchange factor' (ASEF, also called ARHGEF4) ${ }^{4}$. ASEF is a Dbl-family GEF that contains an Src-homology-3 (SH3) domain followed by the Dbl-homology (DH) and pleckstrinhomology $(\mathrm{PH})$ domains characteristic of Dbl-family GEFs that specifically activate members of the Rho family of GTPases. DH and PH domains in Dbl proteins catalyze the exchange of GDP for GTP in Rho GTPases, allowing them to signal to downstream effectors ${ }^{5}$. ASEF is homologous to the Dbl proteins collybistin (also called PEM2) and SPATA13 (also called ASEF2), both of which have been shown to activate specifically CDC42 and not other Rhofamily GTPases 6,7 .

Previous data suggest that ASEF exists in an autoinhibited form and is activated upon binding of $\mathrm{APC}^{\mathrm{Arm}}$ to the region termed the APC-binding region $(\mathrm{ABR})^{8}$. Binding of $\mathrm{APC}^{\mathrm{Arm}}$ to the ABR stimulates activation of the Rho GTPase RAC1 by ASEF. Furthermore, truncation of the ABR is sufficient to relieve autoinhibition, rendering ASEF constitutively active. In addition, truncated, but not wild-type, APC has been shown to efficiently activate ASEF to promote cancer cell migration ${ }^{9}$.

In this study, we wished to delineate the molecular mechanism by which APC relieves autoinhibition of ASEF to stimulate its GEF activity and to assess the contribution of ASEF to $\mathrm{CRC}$ tumorigenesis. To our surprise, and in contrast to previous reports, we found that ASEF specifically activated CDC42, but not RAC1 or other Rho GTPases, in vitro and in vivo. Furthermore, whereas binding of APC ${ }^{\text {Arm }}$ to ASEF stimulated robust exchange activity on $\mathrm{CDC} 42$, truncation of the ABR of ASEF did not activate ASEF. Instead, truncation of the SH3 domain was required to activate the exchange activity of ASEF toward CDC42. Indeed, a 2.25$\AA$-resolution structure of an autoinhibited form of ASEF revealed that the SH3 domain of ASEF forms a tight and extensive interface with both the DH and PH domains to effectively block access of CDC42 to the GTPase-binding site. Also in contrast to previous data ${ }^{9}$, we show that ASEF functions downstream of full-length APC, but not its truncated form, to activate CDC42. Silencing the expression of ASEF in CRC cell lines expressing wild-type, but not truncated, APC resulted in a considerable growth advantage for these cells. Finally, we show that a recently identified mutation in ASEF found in breast cancer abolishes the GEF activity of ASEF. These data suggest that ASEF is a tumor suppressor that links full-length APC to CDC42 and that its inactivation, by mutation of either APC or ASEF, promotes cancer progression.

\section{RESULTS}

\section{ASEF is a RhoGEF specific for CDC42}

Previous structural analyses of RhoGEFs bound to their cognate Rho GTPases provide guidelines for predicting the GTPase specificities of DH domains on the basis of their primary sequences ${ }^{10-14}$. DH domains of RAC1-specific GEFs such as Tiam1 and TRIO typically have isoleucine (Ile1187 in Tiam1) in a position where DH domains of CDC42-specific GEFs, such as Intersectin (ITSN) and PEM2/collybistin, have leucine (Leu1376 in ITSN) (Fig. 1a). ASEF features a leucine (Leu421) at the position equivalent to Ile1187 of Tiam1, suggesting that ASEF might be specific for CDC42. Indeed, a fragment of ASEF encompassing the DH and PH domains catalyzed nucleotide exchange specifically on CDC42 but not RAC1 or RHOA (Fig. 1b). Furthermore, ASEF showed no exchange activity toward other Rho family members, including RHOB, RHOC, RAC2, RAC3, RHOG, TC10, TCL and RHOF (Supplementary Fig. 1 online and data not shown). Moreover, an L421I substitution in the DH/PH fragment of ASEF conferred the capacity to activate RAC1 at the expense of CDC42 (Fig. 1c). These data confirm 
the importance of Leu421 of ASEF in dictating specificity toward CDC42 while prohibiting RAC1 activation.

We assessed the in vivo specificity of ASEF for Rho GTPases by monitoring effects of ASEF expression on the actin cytoskeleton, which undergoes distinct morphological rearrangements in response to the activation of different Rho GTPases ${ }^{15}$. Overexpression of a DH/PH fragment of ASEF fused to yellow fluorescent protein (YFP-ASEF DH/PH) in NIH3T3 mouse fibroblasts resulted in spindle-like projections rich in F-actin, similar to the filopodia induced by activated CDC42 fused to green fluorescent protein (GFP-CDC42 61L) but not the lamella induced by activated RAC1 fused to GFP (GFP-RAC1 61L) (Fig. 2a). The isolated CRIB domain from the CDC42-specific effector N-WASP (N-WASP CRIB) binds to, and blocks signaling downstream of, active CDC42 but not RAC1 in a dominant-negative fashion ${ }^{16}$. Coexpression of N-WASP CRIB with either GFP-CDC42 61L or YFP-ASEF DH/PH effectively suppressed the formation of filopodia. In contrast, N-WASP CRIB did not block lamella formation induced by RAC1 (Fig. 2b). Therefore, in contrast to its original characterization, ASEF specifically activates CDC42 and not other Rho GTPases in vitro and in vivo.

\section{ASEF is autoinhibited by the SH3 domain}

The primary ASEF isoform expressed in human tissues is encoded by ARHGEF4 (ref. ${ }^{17}$; here called ASEF). This isoform contains sequences on the $\mathrm{N}$-terminal side of the ABR domain that are distinct from those encoded in the gene that was originally cloned ${ }^{8}$.We therefore used this predominant isoform throughout the experiments below as full-length ASEF (residues 1-690; see Supplementary Methods.online).

To map regions within ASEF necessary for the autoinhibition of exchange activity, a series of truncated forms of ASEF (Fig. 3a) were tested for their capacity to activate CDC42 in vitro. To our surprise, and in contrast to previous reports 8,9 , truncation of the $\mathrm{N}$ terminus alone $(\triangle \mathrm{N})$ or together with part or all of the $\mathrm{ABR}(\triangle \mathrm{ABR} 1-\triangle \mathrm{ABR} 4)$ did not elicit guanine nucleotide exchange on CDC42 (Fig. 3b). However, further N-terminal truncation to remove the SH3 domain of ASEF (DH/PH) greatly increased the exchange activity of ASEF toward CDC42. We found similar results upon overexpression of N-terminally truncated forms of ASEF in NIH3T3 cells (Fig. 3c). That is, full-length ASEF, as well as N-terminally truncated ASEF lacking its $\mathrm{ABR}$ but retaining its $\mathrm{SH} 3$ domain $(\triangle \mathrm{ABR} 4)$, were incapable of producing $\mathrm{CDC} 42$ like filopodia. In contrast, $\mathrm{ASEF} \mathrm{DH} / \mathrm{PH}$, which lacks both the $\mathrm{ABR}$ and $\mathrm{SH} 3$ domains, induced filopodia formation (Fig. 2a and Fig. 3c). Therefore, the SH3 domain is fully responsible for autoinhibition of the exchange activity of ASEF in vitro and in vivo, and regions on the Nterminal side of the SH3 domain, including the ABR, are irrelevant for this autoinhibition. Attempts to measure increased CDC42-GTP in cells expressing ASEF by affinity precipitation assays were unsuccessful owing to persistent elevated CDC42-GTP levels in the absence of serum (data not shown, and refs. ${ }^{18,19}$ ).

\section{Identification of the core APC-binding motif}

The ABR is a 53-residue region of ASEF necessary for interaction with the armadillo repeats of APC. The ABR is conserved among ASEF orthologs and is approximately 50\% identical in sequence to the ABR of ASEF2. Collybistin does not contain an ABR region ${ }^{6,20}$ and therefore is not regulated by APC (data not shown).

To identify specific functional determinants within the ABR, N-terminally truncated forms of ASEF were tested for their capacity to activate CDC42 in the presence of the full armadillo repeat array of APC. As expected, incubation of ASEF $\triangle \mathrm{N}$ with APC ${ }^{\mathrm{Arm}}$ stimulated the guanine nucleotide exchange activity of ASEF toward CDC42, whereas ASEF $\triangle \mathrm{ABR} 4$, which 
completely lacks the ABR, was not stimulated by APC Arm (Fig. 4a). Furthermore, ASEF $\triangle \mathrm{ABR} 1, \mathrm{ASEF} \triangle \mathrm{ABR} 2$ and ASEF $\triangle \mathrm{ABR} 3$ were activated by APC ${ }^{\text {Arm }}$ similarly to ASEF $\triangle \mathrm{N}$. These results indicate that the minimal ABR sequence (residues 183-193) encompassed in ASEF $\triangle \mathrm{ABR} 3$ and lacking in ASEF $\triangle \mathrm{ABR} 4$ is absolutely required for interaction with $\mathrm{APC}^{\mathrm{Arm}}$ and concomitant activation of $\mathrm{CDC} 42$ by ASEF. This region, which we term the core ATP-binding (CAB) motif, is immediately adjacent to the $\mathrm{SH} 3$ domain on the N-terminal side and is strictly conserved in ASEF2 (Fig. 4b).

The ASEF-related homolog ASEF2 shows high sequence conservation with ASEF, and, like ASEF, its GEF activity is stimulated by APC ${ }^{\text {Arm }}$. However, APC ${ }^{\text {Arm }}$ has been reported to interact with the SH3 domain and not the ABR of ASEF2 to stimulate GEF activity ${ }^{7}$. We found that ASEF $2 \triangle \mathrm{N}$ (containing the ABR) was robustly stimulated by APC ${ }^{\text {Arm }}$ to catalyze nucleotide exchange on CDC42, whereas ASEF2 $\triangle \mathrm{ABR} 4$ (no ABR) was not (Fig. 4c). Therefore, as with ASEF, the ABR region within ASEF2 is essential for activation of RhoGEF catalytic function by APC ${ }^{A r m}$. Given the strict conservation of the CAB motif in both ASEF and ASEF2, it is highly likely that the CAB motif of ASEF2 is absolutely required for binding to APC.

\section{Full-length but not truncated APC activates ASEF}

APC mutants associated with CRCs lack binding sites for microtubules and $\beta$-catenin but retain the oligomerization domain and the highly conserved ARM repeats (residues 335-735) ${ }^{21}$ (Fig. 5a). To directly assess the capacity of APC to activate ASEF, we expressed these proteins in COS-7 cells, where the subcellular localization of exogenous full-length or truncated forms of APC recapitulates their endogenous localization in CRC cell lines ${ }^{22,23}$. Of note, in COS-7 cells, overexpression of activated CDC42 (GFP-CDC42 61L) results in the formation of dorsal and peripheral membrane ruffles, whereas activated RAC1 in COS-7 cells induces peripheral ruffles that are thicker and morphologically distinct ${ }^{24}$ (Fig. 5b). Consistent with our previous data, activated ASEF (YFP-ASEF DH/PH) induces membrane ruffles in COS-7 cells indistinguishable from those induced by constitutively active CDC42.

When expressed separately, both GFP-tagged APC ${ }^{\text {Arm }}$ and mCherry-tagged full-length (FL) ASEF showed diffuse nuclear and cytoplasmic localization (Fig. 5c) with no obvious changes in cell morphology compared to cells expressing mCherry alone. However, coexpression of GFP-APC ${ }^{\text {Arm }}$ and mCherry-FL ASEF resulted in their colocalization in prominent peripheral (arrow) and dorsal (arrow-head) ruffles. Colocalization and ruffle formation were abrogated if the expressed APC ${ }^{\text {Arm }}$ protein harbored a point mutation (N507K; Fig. 5c) previously shown to prevent interaction of APC with ASEF (Supplementary Fig. 2 online and ref. ${ }^{25}$ ).

Furthermore, consistent with our in vitro data, concomitant colocalization and ruffle formation were lost when $\mathrm{APC}^{\mathrm{Arm}}$ was coexpressed with a version of ASEF lacking the ABR (mCherryASEF $\triangle \mathrm{ABR} 4)$.

Nonsense mutation of codon 1061 results in a common germline truncation of APC associated with $\mathrm{CRCs}^{2}$ (Fig. 5a). In agreement with published reports ${ }^{22,26}$, exogenously expressed GFPtagged full-length APC localized to a filamentous meshwork likely to be the microtubule cytoskeleton, whereas a similar construct of APC terminating at position 1061 (APC 1061) was distributed diffusely and in intermittent dense puncta throughout the cytoplasm (Fig. 5d). Notably, when GFP-FL APC was coexpressed with mCherry-FL ASEF, the two proteins colocalized in dorsal ruffles and in puncta close to the plasma membrane (Fig. 5d, inset), suggesting that they interact to activate CDC42. Furthermore, the N507K mutation in the context of full-length APC (GFP-FL APC N507K) disrupted colocalization with mCherryFL ASEF, and no dorsal ruffles were observed. Contrary to the previous suggestion that truncated APC activates ASEF in vivo ${ }^{9}$, GFP-APC 1061 did not colocalize with mCherry-FL 
ASEF or promote membrane ruffling. Therefore, we conclude that full-length APC, but not a form terminating at residue 1061, readily stimulates ASEF in COS-7 cells.

\section{Structure of autoinhibited ASEF}

ASEF $\triangle \mathrm{ABR} 2$ is a minimal fragment of ASEF that is completely inhibited in the absence of APC (Fig. 3b) yet fully activated by APC Arm (Fig. 4a). To elucidate the structural basis for the autoinhibition of ASEF and its related activation by APC, we determined a high-resolution structure of ASEF $\triangle \mathrm{ABR} 2$ (residues 170-632) by X-ray crystallography (Supplementary Fig. 3 online).

At the heart of the structure is the SH3 domain, which organizes the inhibited conformation of ASEF (Fig. 6a). The five $\beta$-strands of the ASEF SH3 domain form a $\beta$-barrel similar to other SH3 domains. Other common features include the RT loop (between $\beta 1$ and $\beta 2$ ) and the n-Src loop (between $\beta 2$ and $\beta 3$ ), which typically engage peptide ligands containing multiple prolines ${ }^{27}$, as well as a short $3{ }_{10}$-helix separating $\beta 4$ and $\beta 5$. Capping the end of the $\beta$-barrel is the $\mathrm{CAB}$ motif, which forms an extended region (residues 183-186) and a short $\alpha$-helix $(\alpha \mathrm{CAB}$, residues 187-193). $\alpha \mathrm{CAB}$ within the $\mathrm{CAB}$ motif primarily interacts with the $\mathrm{SH} 3$ domain through contacts with $\beta 2$ and the n-Src loop. Residues Glu183, Leu185, Glu189 and Asp193 within the CAB motif are solvent exposed and evolutionarily conserved among ASEF homologs, indicating that they may be important for interaction with APC.

The SH3 domain forms an extensive interface primarily with the DH domain, but major interactions also include the PH domain. Of the $4,170 \AA^{2}$ of solvent-accessible area on the surface of the ASEF SH3 domain, $1170 \AA^{2}$ are buried through interaction with the DH and PH domains. Elements from the SH3 domain, including $\beta 1$, the RT loop, the $3_{10}$-helix, $\beta 5$ and the extended region on the $\mathrm{C}$-terminal side of $\beta 5$, form multiple polar and nonpolar interactions with $\alpha 1, \alpha 5$ and $\alpha 6$ of the DH domain. This region of the DH domain is the conserved surface that is responsible for binding to the switch regions of nucleotide-free Rho GTPases, as required for their activation by catalyzed guanine nucleotide exchange ${ }^{10}$. Therefore, in this form, the SH3 domain would block access of CDC42 to the DH domain of ASEF, providing the structural basis for the autoinhibition of ASEF by its SH3 domain as described above. Important interactions are also observed between the $\mathrm{SH} 3$ domain and the $\mathrm{PH}$ domain, occurring between the RT loop of the SH 3 domain and $\beta 1$ and $\beta 2$ of the PH domain and between the $\beta 3-\beta 4$ loop of the SH3 domain and $\alpha \mathrm{C}$ of the PH domain. Helix $\alpha 6$ of the DH domain is extended and similar in length to $\alpha 6$ in ITSN and Tiam1 (refs. ${ }^{10,14}$ ) but adopts a unique arched conformation not observed in other DH domains. The arch allows $\alpha 6$ to conform to the surface of the SH3 domain, and this configuration is stabilized by the extensive interactions between the $\mathrm{SH} 3$ domain, $\alpha 6$ and the PH domain. This arrangement suggests that in the absence of the SH3 domain - for example, in truncated variants or upon movement of the domain by engagement of APC $-\alpha 6$ will adopt an alternative conformation.

Although the SH3 domain of ASEF forms a unique interface with DH and PH domains that is distinct from those observed between other SH3 domains and polyproline (PXXP)-containing peptides, the PXXP recognition surface is partially occluded (see Supplementary Fig. 4 online). Therefore, in the observed orientation, the binding of PXXP sequences to the SH3 domain remains energetically unfavorable. An in-depth description of the PXXP-binding site of ASEF is available in the Supplementary Data and Supplementary Discussion online.

\section{Comparison of ASEF with collybistin DH/PH-CDC42}

Collybistin is a Dbl-family RhoGEF that specifically activates CDC42 (ref. ${ }^{6}$ ) and is highly similar to ASEF; their respective DH and PH domains are 68\% identical. A recent structure encompassing the DH/PH cassette of collybistin bound to nucleotide-depleted CDC42 (ref. 
${ }^{28}$ ) highlights considerable repositioning of the $\alpha 6$ helix of its DH domain, relative to the structure of ASEF described here (Fig. 6b). In free ASEF, $\alpha 6$ curves toward the main body of the DH domain and is secured in this conformation by the SH3 domain. Modeling CDC42 from the structure of collybistin-CDC42 onto the GTPase-binding site of ASEF reveals that the DH and PH domains of ASEF would prohibit CDC42 binding and exchange irrespective of the SH3 domain. In this inhibited conformation, $\alpha 6$ would impinge upon switch 2 of CDC42, and regions in the PH domain would clash with $\alpha 3 \mathrm{a}, \alpha 3 \mathrm{~b}$ and $\alpha 4$ of CDC42. To relieve this steric clash, the DH and PH domains of ASEF must adopt a more open conformation, probably one similar to that observed in collybistin-CDC42. In doing so, $\alpha 6$ must rotate $\sim 45^{\circ}$, to a position more parallel to the main body of the DH domain, to facilitate an $\sim 30$ - $\AA$ translation of the PH domain. Molecular dynamics simulations of ASEF have also suggested conformational flexibility between the $\mathrm{DH}$ and $\mathrm{PH}$ domains ${ }^{29}$. Additionally, in the structure of collybistin-CDC42, the region on the C-terminal side of $\alpha \mathrm{C}$ ( $\alpha \mathrm{C}$ extension) becomes ordered and packs against the $\mathrm{C}$ terminus of $\alpha \mathrm{C}$, presumably to stabilize this conformation. Although the ASEF $\triangle \mathrm{ABR} 2$ construct used for crystallization encompasses the equivalent $\alpha \mathrm{C}$ extension, electron density in this region is missing from the structure.

From these observations, a mechanism of ASEF activation emerges in which binding of the ABR of ASEF by APC retracts the SH3 domain from the DH and PH domains, allowing the GEF to relax into an open and active conformation. Previously determined structures of DH and $\mathrm{PH}$ domains have intimated plasticity of $\alpha 6$, especially in the $\mathrm{DH}$ and $\mathrm{PH}$ domains of Sos1 $\left(\right.$ ref. ${ }^{30}$ ). The present comparison of ASEF and collybistin structures reveals large domain rearrangements of $\mathrm{DH} / \mathrm{PH}$ cassettes required for regulating the autoinhibition and subsequent activation of Dbl-family GEFs.

\section{Interface of the SH3 domain with the $\mathrm{DH}$ and $\mathrm{PH}$ domains}

The DH and PH domains of ASEF form an arch stabilized at its center by the SH3 domain (Fig. 7a). Two sets of hydrophobic interactions in the crown of the arch are crucial for stabilizing the interface. Chief among these are the interactions of Trp203 at the base of the RT loop of the SH3 domain, which act as the keystone by packing into a central hydrophobic pocket formed by residues in $\alpha 5$ and $\alpha 6$ of the DH domain. A hydrogen bond between the indole nitrogen of Trp203 and the sulfhydryl group of Cys428 cements the interactions. Also within the RT loop, Met208 inserts into a second hydrophobic pocket framed by residues from $\alpha 6$ of the DH domain and $\beta 1$ and $\beta 2$ of the PH domain, and these interactions seem to be crucial for maintenance of the arched conformation of the DH and PH domains. In between Trp203 and Met208 of the RT loop, Asp204 forms hydrogen bonds with the side chain of Asn466 within $\alpha 6$, and the backbone oxygen of Val206 interacts with the side chain of Lys 477 to further stabilize the arch. Asn466 is highly conserved in DH domains, where it is required to bind switch 2 of Rho GTPases during nucleotide exchange ${ }^{5}$; thus, its central position within the interface with the SH3 domain emphasizes the overlap between binding sites in the DH domain for the SH3 domain and for CDC42.

Bracketing Trp203 and Met208 are sets of hydrophilic interactions that further stabilize the arched conformation of the DH and PH domains. For instance, Asp210 in the RT loop forms a salt bridge with Lys533 in $\beta 3$ of the PH domain, and Asp238 in the $\beta 3-\beta 4$ loop of the SH3 domain binds Arg596 in $\alpha \mathrm{C}$ of the PH domain. On the opposite end of the arch and juxtaposed to the ABR, a network of hydrogen bonds centered on Glu436 in $\alpha 5$ of the DH domain stabilizes this portion of the interface. Arg249 in $\beta 5$ of the SH3 domain is particularly important; its side chain inserts between Glu436 and Glu291 in $\alpha 1$ to form an electrostatic bridge between the three residues. Of note, Glu291 is also crucial to the GEF activity of DH domains, as it is needed to form conserved interactions with switch 1 of Rho GTPases ${ }^{5}$. In addition to interactions with Arg249, Glu436 also forms hydrogen bonds to the backbone atoms of Val252 
and Asn253, on the C-terminal side of $\beta 5$ of the SH3 domain. The side chain of Asn253 forms important interactions with the side chain of Asn287 in $\alpha 1$ of the DH domain to secure this end of the interface. Because this network of hydrophilic interactions is adjacent to the CAB region within the ABR, we suggest that these interactions are the first to be disrupted upon APC binding and that this disruption is followed by 'unzipping' of the remainder of the interface to activate ASEF.

We mutated residues in the SH3 domain that interact with the DH and PH domains of ASEF to alanines, with the anticipation that disrupting this interface might relieve the autoinhibition of ASEF. Individual substitutions were made in the background of fully inhibited ASEF $\triangle \mathrm{ABR} 2$, and mutant proteins were tested for GEF activity in vitro (Fig. 7b). Mutation of residues that form interactions with the main body of the DH domain (Trp203, Arg249 and Asn253) were most effective at relieving inhibition, especially W203A, which restored the full GEF activity of ASEF toward CDC42 in vitro. The equivalent mutation (W156A) in ASEF2 $\triangle \mathrm{N}$ is also activating (Fig. 7b, right graph), indicating that ASEF and ASEF2 are similarly auto-inhibited by their respective SH3 domains. Consistent with these in vitro results, ASEF $\triangle \mathrm{ABR} 4 \mathrm{~W} 203 \mathrm{~A}$ and a constitutively active ASEF DH/PH protein induced filopodia formation to a similar degree in NIH3T3 cells (Fig. 7c).

\section{ASEF is a candidate tumor suppressor}

To better understand the regulation of ASEF by APC in CRCs, we suppressed the expression of ASEF by RNA interference in CRC cell lines that retain full-length (SW48 and HCT116) or truncated (SW480) APC ${ }^{31,32}$ and assessed CDC42 activation and tumorigenic potential. We established individual cell lines stably expressing each of two independent short hairpin RNAs (shRNA-1 and shRNA-2) targeting $A S E F$ or a nontargeting control (shRNA-C) and assessed the amount of GTP-bound CDC42 by affinity precipitation. Knockdown of ASEF messenger RNA in SW48 and HCT116 cell lines harboring full-length APC led to decreased levels of CDC42-GTP (Fig. 8a). In contrast, the amount of CDC42-GTP remained unchanged in SW480 cells despite depletion of ASEF mRNA.

The HCT116 and SW480 cell lines expressing shRNAs were also assessed for anchorageindependent growth in soft agar (Fig. 8b; SW48 cells grow poorly in soft agar). HCT116 cells expressing $A S E F$-specific shRNAs showed a two- to four-fold increase in anchorageindependent growth compared to cells expressing control shRNA, whereas colony growth of SW480 cells was not affected by ASEF knockdown. Notably, in both cell types, anchorageindependent growth was promoted by expression of a dominant-negative form of CDC42 fused to a hemag-glutinin tag (HA-CDC42 17N), which forms a nonproductive complex with CDC42-specific GEFs and suppresses their activity ${ }^{33}$. These data suggest that full-length APC promotes the activation of CDC42 by ASEF to suppress the anchorage-independent growth of HCT 116 cells. In SW480 cells expressing truncated APC that does not activate ASEF, CDC42GTP levels remained high and no enhancement of anchorage-independent growth was observed upon $A S E F$ knockdown.

ASEF has recently been found to be mutated in human cancers ${ }^{34}$ and designated as a candidate cancer gene predicted to have higher-than-background mutation frequencies in cancers. Two separate missense mutations of ASEF have been uncovered in breast cancers: K100R in the N terminus and T441R in the DH domain. In our structure of ASEF, Thr441 resides in the turn separating $\alpha 5$ and $\alpha 6$, where it participates in an extensive network of hydrogen bonds with residues in $\alpha 1, \alpha 3, \alpha 5$ and $\alpha 6$ to stabilize the DH domain fold (Fig. 8c). Substitution of arginine for Thr441 would disrupt these interactions and would probably be very detrimental to the overall fold and associated exchange activity of the DH domain. Consistent with this assumption, T441R mutation in the constitutively active ASEF DH/PH protein abolished its ability to promote ruffling in COS-7 cells and abolished the activation of ASEF $\triangle \mathrm{ABR} 2$ by 


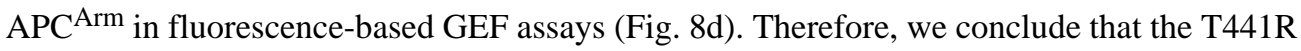
mutation in ASEF causes loss of the GEF activity of ASEF and thereby leads to loss of regulation of CDC42 by APC. Together, these data imply that loss of ASEF function is advantageous to tumor progression and that ASEF acts as a tumor suppressor.

\section{DISCUSSION}

There are approximately 70 Dbl-family RhoGEFs that differentially activate 20 Rho GTPases in humans. Although the ASEF-related GEFs collybistin ${ }^{6,28}$ and ASEF2 (ref. ${ }^{7}$ ) are specific for CDC42, the specificity of ASEF for Rho GTPases has been controversial. Initial reports indicated that ASEF was a RAC1-specific $\mathrm{GEF}^{8,9,29}$, whereas a recent study ${ }^{18}$ showed that ASEF was specific for CDC42 and not RAC1. Consistent with that study, our data demonstrate that ASEF acts as a CDC42 GEF in vitro and in vivo and does not activate other Rho GTPases. Notably, knockdown of ASEF in CRC cell lines expressing full-length APC decreases the levels of GTP-bound CDC42. This clarification of ASEF specificity is biologically relevant, as $\mathrm{RAC} 1$ and $\mathrm{CDC} 42$ regulate distinct cellular processes ${ }^{35}$.

The structure of autoinhibited ASEF reveals that the SH3 domain forms a unique interface with regions in both the DH and PH domains and masks the surface on the DH domain $(\alpha 1, \alpha 5$ and a6) required for binding Rho GTPases during nucleotide exchange. This steric occlusion inhibits the GEF activity of ASEF. The CAB motif and the remainder of the ABR are largely solvent exposed and not part of the inhibitory interface. Therefore, truncated forms of ASEF that lack the ABR but retain the SH3 domain are constitutively inhibited, and previous studies that have used such forms of ASEF and assumed their constitutive activation should be reinterpreted. Mutations in the SH3 domain (R249A and N253A) that enhance the exchange activity of full-length ASEF without perturbing the capacity of the SH3 domain to bind polyproline-containing ligands could be used in place of truncated forms of ASEF in future studies.

Recently, a different mechanism for ASEF2 autoinhibition has been put forth, in which interactions between the $\mathrm{ABR}$ and $\mathrm{SH} 3$ domains and regions on the C-terminal side of the $\mathrm{PH}$ domain are important for its autoinhibition ${ }^{7}$. However, for both ASEF and ASEF2, we find that removal of the $\mathrm{C}$-terminal region following the $\mathrm{PH}$ domain does not influence the exchange potential of these GEFs (see Supplementary Fig. 5 online). Furthermore, substitutions in the SH3 domain of ASEF designed to disrupt its interaction with the DH and PH domains, as well as analogous substitutions in ASEF2, activate the respective exchange potentials of these GEFs, indicating that our proposed mechanism of autoinhibition of ASEF is almost certainly conserved in ASEF2.

It is becoming clear that APC is involved in the regulation of several cellular processes independent of its role in Wnt signaling, including the reorganization and stabilization of the cytoskeleton and regulation of cell migration, cell division and apoptosis ${ }^{4}$. It is increasingly appreciated that truncated forms of APC associated with cancers might have differential activities that promote tumorigenesis independently of the loss of $\beta$-catenin regulation. ASEF is reported to have a crucial role in cellular migration downstream of truncated $\mathrm{APC}^{9}$. We show here that truncation of APC leads to the loss of ASEF function. Truncated APC does not promote morphological changes associated with ASEF and CDC42 activation, probably owing to the altered cellular localization of truncated APC. The coupling of APC to CDC42 activation via ASEF that we observed in CRC cell lines expressing full-length APC is lost upon APC truncation. Moreover, suppression of ASEF in CRC cells expressing full-length APC greatly increases anchorage-independent growth of these cells, whereas colony growth is not affected by ASEF knockdown in cells expressing truncated APC. These data support a mechanism in which ASEF signals downstream of full-length APC to activate CDC42 to suppress anchorage- 
independent growth. Notably, dominant-negative CDC42 enhances anchorage-independent growth of CRC cell lines expressing either full-length or truncated APC, demonstrating that the general loss of CDC42 activity by sequestration of GEFs can promote CRC.

ASEF has recently been found to be mutated in human breast cancers and not in $\mathrm{CRCs}^{34}$. In one breast tumor, ASEF acquired a homozygous mutation at codon $441(\mathrm{~T} 441 \mathrm{R})^{36}$ that abolishes catalytic activity (Fig. 8d), suggesting that ASEF acts as a tumor suppressor in breast epithelial cells. Together with our findings in CRC cell lines, these data strongly imply that the loss of ASEF function is advantageous to tumor progression in general and that ASEF is a tumor suppressor in multiple cell types. Given that APC is not typically mutated in breast cancers, and ASEF mutations have not been found in CRCs, it is possible that loss of ASEF function can be achieved indirectly through mutation of APC (in CRCs) or by direct mutation and inactivation of ASEF (in breast cancers). The consequence of regulation of CDC42 by $\mathrm{APC}$ and ASEF and the role of CDC42 itself in cancer progression are not known. Future studies will determine the signaling pathways mediated by ASEF and uncover the roles of ASEF and CDC42 in human cancers.

\section{METHODS}

\section{Protein expression and purification}

His $_{6}$-tagged ASEF and ASEF2 (human) proteins were expressed in Rosetta BL21(DE3) Escherichia coli cells. To produce selenomethionine (SeMet)-substituted ASEF $\triangle$ ABR2 for structure determination, ASEF $\triangle \mathrm{ABR} 2$ was expressed using the methionine-auxotrophic $E$. coli strain B834(DE3) and cultured in L-SeMet-enriched media using methods similar to those described ${ }^{37}$. Detailed descriptions of protein expression and purification are available in the Supplementary Methods. APC Arm proteins (human, residues 309-798) were purified exactly as described for ASEF proteins, except all buffers contained $500 \mathrm{mM} \mathrm{NaCl}$ and EDTA was not included in buffer B.

Human placental CDC42 (residues 1-188; C188S), RAC1 (residues 1-189; C189S) and RHOA (residues 1-190; C190S) were expressed in E. coli strain BL21(DE3) and purified as described $^{13}$. His 6 -tagged Rho GTPases used in Supplementary Figure 1 were prepared as described $^{38}$.

\section{Guanine nucleotide exchange assays}

$N$-methylanthraniloyl (mant)-GTP incorporation into bacterially purified Rho GTPases was carried out with a Perkin-Elmer LS 55 spectrometer at $20^{\circ} \mathrm{C}$. Exchange-reaction assay mixtures containing $20 \mathrm{mM}$ Tris (pH 7.5), $100 \mathrm{mM} \mathrm{NaCl}, 5 \mathrm{mM} \mathrm{MgCl}_{2}, 1 \mathrm{mM}$ DTT, $5 \%$ (v/v) glycerol, $400 \mathrm{nM}$ mant-GTP (Molecular Probes) and $2 \mu \mathrm{M}$ of the relevant GTPase were prepared and allowed to equilibrate with continuous stirring. After equilibration, ASEF or ASEF2 proteins were added at $1 \mu \mathrm{M}$, and the relative fluorescence $\left(\lambda_{\text {emission }}=360 \mathrm{~nm}, \lambda_{\text {excitation }}=440 \mathrm{~nm}\right)$ was monitored. For experiments including APC ${ }^{\text {Arm }}$, we incubated ASEF or ASEF2 proteins on ice with $\mathrm{APC}^{\mathrm{Arm}}$ for $10 \mathrm{~min}$ before adding them to GEF reactions. The final concentration of $\mathrm{APC}^{\mathrm{Arm}}$ in GEF reactions was $1 \mu \mathrm{M}$.

\section{Immunostaining and fluorescence microscopy}

For live-cell microscopy, NIH3T3 and COS-7 cells were plated, transfected and imaged in 35$\mathrm{mm}$ MatTek culture dishes. Plates were mounted 16-24 h after transfection on a temperaturecontrolled heated stage (TempControl 37-2 digital, Carl Zeiss MicroImaging), and live cells were examined with an inverted laser scanning confocal microscope (Zeiss 510 LSM) using an oil-immersion $\times 63$ NA 1.4 objective. For studies of mCherry-ASEF colocalization with GFP-APC, images were captured by sequential scanning with the 488-nM spectral line of an 
argon ion laser and the 543-nM HeNe1 laser and emission filters BP 505-530 (for GFP) and LP 585 (for mCherry).

For indirect immunofluorescence, NIH3T3 cells were plated and transiently transfected in fourwell Lab-Tek chambered glass coverslides (Nunc). The next day, cells were fixed with $4 \%$ (v/ v) paraformaldehyde, permeabilized with $0.2 \%$ (v/v) Triton X-100 and stained with Alexa 594phalloidin (Molecular Probes). Cells were mounted with FluoroSave (Calbiochem) and examined with a Zeiss 510 LSM confocal microscope. Digital images were processed and brightness and contrast adjusted with Adobe Photoshop CS2.

\section{Crystallization of ASEF}

SeMet-substituted ASEF $\triangle \mathrm{ABR} 2$ was crystallized by vapor diffusion in sitting drops. Drops were formed by a 2:1 ratio of protein solution $\left(\sim 9 \mathrm{mg} \mathrm{ml}^{-1}\right.$ in buffer B) to reservoir solution (100 mM HEPES (pH 7), 20\% (w/v) PEG 3,350, $200 \mathrm{mM}$ magnesium acetate and 10\% (v/v) glycerol), then equilibrated against $1 \mathrm{ml}$ of reservoir solution at $18{ }^{\circ} \mathrm{C}$. Reproducibility and crystal quality were generally improved by microcrystal seeding. For data collection at $100 \mathrm{~K}$, crystals were suspended in a rayon loop (Hampton Research) and snap-frozen by immersion into liquid nitrogen. Crystals belong to the space group $\mathrm{C} 2$, with unit cell dimensions of $a=$ 116.4, $b=80.5, c=101.5$ and $\beta=104.5^{\circ}$. There are two apparent monomers in the asymmetric unit. A three-wavelength MAD data set $\left(\lambda_{1}=12,661.14 \mathrm{eV}, \lambda_{2}=12,659.14 \mathrm{eV}, \lambda_{3}=12,760.14\right.$ eV) was collected on a single crystal on the Southeast Regional Collaborative Access Team (SER-CAT) beamline 22-ID at the Advanced Photon Source of Argonne National Laboratory.

A native data set was subsequently collected at the University of North Carolina X-ray facility on a single crystal at $100 \mathrm{~K}$ using an R-AXIS IV++ area detector and a Rigaku RU-H3R rotating anode X-ray source equipped with focusing optics. Crystals were produced from native protein as described for SeMet-substituted ASEF $\triangle \mathrm{ABR} 2$, then incubated in a soaking solution containing $100 \mathrm{mM}$ HEPES (pH 7), 50\% (w/v) PEG 3,350, $200 \mathrm{mM}$ magnesium acetate and $10 \%(\mathrm{v} / \mathrm{v})$ glycerol. Soaked crystals had unit cell dimensions of $a=100.4, b=79.9, c=67.4$ and $\beta=123.0^{\circ}$, with one molecule in the asymmetric unit. All data were processed and scaled using HKL2000 (ref. ${ }^{39}$ ).

\section{Model building and refinement}

The Patterson peak-searching program SHELXD ${ }^{40}$ and the three-wavelength MAD dataset were initially used to determine the positions of two sets of eight selenium atoms related by two-fold noncrystallo-graphic symmetry. The positions of the heavy atom sites were refined using the CCP4 program MLPHARE ${ }^{41}$ before phase improvement with solvent flattening, histogram matching and noncrystallographic symmetry averaging using $\mathrm{DM}^{42}$ The resulting electron density map was used for initial model building; however, density for the PH domain was almost entirely missing and further refinement gave poor statistics $\left(R_{\text {free }}>35\right)$. Data collected on the native crystal were phased by molecular replacement with the CCP4 program PHASER $^{43}$ using the best monomer model that resulted from MAD experiments. The resulting density map was of high quality and easily interpretable (Supplementary Fig. 3). The structure of ASEF $\triangle \mathrm{ABR} 2$ was completed using native protein data and iterative cycles of model building and TLS refinement with $\mathrm{COOT}^{44}$ and the CCP4 program REFMAC ${ }^{45}$. The final model has a crystallographic $R$-value of $21.7 \%\left(R_{\text {free }}=27.9 \%\right)$ using all data from $15-2.25 \AA$ (see Table 1) and includes residues 181-254 and 278-611 of ASEF. Electron density was uninterpretable for residues 170-180 (N terminus), 255-277 (SH3-DH linker) and 612-632 (C terminus) and therefore could not be modeled. Owing to the poor electron density in this region, residues $561-570$ (in the $\beta 5-\beta 6$ loop of the PH domain) were modeled as glycines. In the Ramachandran plot, $91.8 \%$ of residues are in the most favored regions, $7.3 \%$ are in the 
allowed region and $0.8 \%$ are in the disallowed region. All molecular graphics were generated with PyMOL (http://pymol.sourceforge.net).

\section{CDC42 affinity precipitation assays}

SW48, SW480 and HCT116 cells stably expressing control or ASEF-specific shRNAs were lysed in lysis buffer (50 mM Tris (pH 7.5), $150 \mathrm{mM} \mathrm{NaCl}, 10 \mathrm{mM} \mathrm{MgCl}_{2}, 1 \%$ (v/v) Nonidet $\mathrm{P}-40,0.25 \%(\mathrm{w} / \mathrm{v})$ sodium deoxycholate and $10 \%(\mathrm{v} / \mathrm{v})$ glycerol) plus protease inhibitor cocktail (Roche). Glutathione $S$-transferase (GST)-PAK RBD (see Acknowledgments) immobilized on glutathione-Sepharose $4 \mathrm{~B}$ beads was added to clarified total cell lysates and incubated at $4{ }^{\circ} \mathrm{C}$ for $45 \mathrm{~min}$. Beads were pelleted and washed once with lysis buffer and twice with wash buffer ( $25 \mathrm{mM}$ Tris ( $\mathrm{pH} 7.5$ ), $40 \mathrm{mM} \mathrm{NaCl}, 30 \mathrm{mM} \mathrm{MgCl}_{2}$ ). Total lysate and affinityprecipitated lysate samples were subjected to SDS-PAGE and analyzed by western blotting using CDC42-specific antibodies (Santa Cruz Biotechnology).

\section{Statistical analysis}

Data was obtained from three independent experiments done in triplicate and $P$-values were calculated using a one-tailed Student's $t$-test. Significance for all tests was assumed at $P<$ 0.001 (confidence interval 95\%).

\section{Additional methods}

A detailed description of expression and RNA interference constructs, maintenance of cell lines, reverse-transcription PCR and soft-agar assays is available in the Supplementary Methods.

\section{Supplementary Material}

Refer to Web version on PubMed Central for supplementary material.

\section{ACKNOWLEDGMENTS}

We thank D. Thiel and L. Bauer for technical assistance, Keith Burridge, (University of North Carolina) for the gift of GST-PAK RBD, the M. Hooker imaging facility at the University of North Carolina, Chapel Hill, and the staff at the SER-CAT beamlines for help with data collection. Use of the Advanced Photon Source was supported by the US Department of Energy, Office of Science, Office of Basic Energy Sciences, under contract no. W-31-109-Eng-38. J.S. is supported by grants GM65533 and GM62299 from the US National Institutes of Health. C.J.D. is supported by grant CA063071 from the US National Institutes of Health. K.L.R. is supported by postdoctoral fellowship grant PF-05-129-01(GMC) from the American Cancer Society.

\section{References}

1. Fodde R, Smits R, Clevers H. APC, signal transduction and genetic instability in colorectal cancer. Nat. Rev. Cancer 2001;1:55-67. [PubMed: 11900252]

2. Polakis P. The adenomatous polyposis coli (APC) tumor suppressor. Biochim. Biophys. Acta 1997;1332:F127-F147. [PubMed: 9196022]

3. Segditsas S, Tomlinson I. Colorectal cancer and genetic alterations in the Wnt pathway. Oncogene 2006;25:7531-7537. [PubMed: 17143297]

4. Hanson CA, Miller JR. Non-traditional roles for the Adenomatous Polyposis Coli (APC) tumor suppressor protein. Gene 2005;361:1-12. [PubMed: 16185824]

5. Rossman KL, Der CJ, Sondek J. GEF means go: turning on RHO GTPases with guanine nucleotideexchange factors. Nat. Rev. Mol. Cell Biol 2005;6:167-180. [PubMed: 15688002]

6. Reid T, Bathoorn A, Ahmadian MR, Collard JG. Identification and characterization of hPEM-2, a guanine nucleotide exchange factor specific for CDC42. J. Biol. Chem 1999;274:33587-33593. [PubMed: 10559246] 
7. Hamann MJ, Lubking CM, Luchini DN, Billadeau DD. Asef2 functions as a CDC42 exchange factor and is stimulated by the release of an autoinhibitory module from a concealed C-terminal activation element. Mol. Cell. Biol 2007;27:1380-1393. [PubMed: 17145773]

8. Kawasaki Y, et al. Asef, a link between the tumor suppressor APC and G-protein signaling. Science 2000;289:1194-1197. [PubMed: 10947987]

9. Kawasaki Y, Sato R, Akiyama T. Mutated APC and Asef are involved in the migration of colorectal tumour cells. Nat. Cell Biol 2003;5:211-215. [PubMed: 12598901]

10. Worthylake DK, Rossman KL, Sondek J. Crystal structure of RAC1 in complex with the guanine nucleotide exchange region of Tiam1. Nature 2000;408:682-688. [PubMed: 11130063]

11. Gao Y, Xing J, Streuli M, Leto TL, Zheng Y. Trp(56) of rac1 specifies interaction with a subset of guanine nucleotide exchange factors. J. Biol. Chem 2001;276:47530-47541. [PubMed: 11595749]

12. Karnoub AE, et al. Molecular basis for RAC1 recognition by guanine nucleotide exchange factors. Nat. Struct. Biol 2001;8:1037-1041. [PubMed: 11685227]

13. Cheng L, et al. RhoGEF specificity mutants implicate RhoA as a target for Dbs transforming activity. Mol. Cell. Biol 2002;22:6895-6905. [PubMed: 12215546]

14. Snyder JT, et al. Structural basis for the selective activation of Rho GTPases by Dbl exchange factors. Nat. Struct. Biol 2002;9:468-475. [PubMed: 12006984]

15. Nobes CD, Hall A. Rho, rac, and cdc42 GTPases regulate the assembly of multimolecular focal complexes associated with actin stress fibers, lamellipodia, and filopodia. Cell 1995;81:53-62. [PubMed: 7536630]

16. Nobes CD, Hall A. Rho GTPases control polarity, protrusion, and adhesion during cell movement. J. Cell Biol 1999;144:1235-1244. [PubMed: 10087266]

17. Thiesen S, Kubart S, Ropers HH, Nothwang HG. Isolation of two novel human RhoGEFs, ARHGEF3 and ARHGEF4, in 3p13-21 and 2q22. Biochem. Biophys. Res. Commun 2000;273:364-369. [PubMed: 10873612]

18. Gotthardt K, Ahmadian MR. Asef is a CDC42-specific guanine nucleotide exchange factor. Biol. Chem 2007;388:67-71. [PubMed: 17214551]

19. Nalbant P, Hodgson L, Kraynov V, Toutchkine A, Hahn KM. Activation of endogenous CDC42 visualized in living cells. Science 2004;305:1615-1619. [PubMed: 15361624]

20. Kins S, Betz H, Kirsch J. Collybistin, a newly identified brain-specific GEF, induces submembrane clustering of gephyrin. Nat. Neurosci 2000;3:22-29. [PubMed: 10607391]

21. Nathke I. Cytoskeleton out of the cupboard: colon cancer and cytoskeletal changes induced by loss of APC. Nat. Rev. Cancer 2006;6:967-974. [PubMed: 17093505]

22. Munemitsu S, et al. The APC gene product associates with microtubules in vivo and promotes their assembly in vitro. Cancer Res 1994;54:3676-3681. [PubMed: 8033083]

23. Rosin-Arbesfeld R, Ihrke G, Bienz M. Actin-dependent membrane association of the APC tumour suppressor in polarized mammalian epithelial cells. EMBO J 2001;20:5929-5939. [PubMed: 11689433]

24. Kurokawa K, Itoh RE, Yoshizaki H, Nakamura YO, Matsuda M. Coactivation of RAC1 and CDC42 at lamellipodia and membrane ruffles induced by epidermal growth factor. Mol. Biol. Cell 2004;15:1003-1010. [PubMed: 14699061]

25. Watanabe T, et al. Interaction with IQGAP1 links APC to RAC1, CDC42, and actin filaments during cell polarization and migration. Dev. Cell 2004;7:871-883. [PubMed: 15572129]

26. Smith KJ, et al. Wild-type but not mutant APC associates with the microtubule cytoskeleton. Cancer Res 1994;54:3672-3675. [PubMed: 8033082]

27. Li SS. Specificity and versatility of SH3 and other proline-recognition domains: structural basis and implications for cellular signal transduction. Biochem. J 2005;390:641-653. [PubMed: 16134966]

28. Xiang S, et al. The crystal structure of CDC42 in complex with collybistin II, a gephyrin-interacting guanine nucleotide exchange factor. J. Mol. Biol 2006;359:35-46. [PubMed: 16616186]

29. Murayama K, et al. Crystal structure of the rac activator, Asef, reveals its autoinhibitory mechanism. J. Biol. Chem 2007;282:4238-4242. [PubMed: 17190834] 
30. Soisson SM, Nimnual AS, Uy M, Bar-Sagi D, Kuriyan J. Crystal structure of the Dbl and pleckstrin homology domains from the human Son of sevenless protein. Cell 1998;95:259-268. [PubMed: 9790532]

31. Morin PJ, et al. Activation of beta-catenin-Tcf signaling in colon cancer by mutations in beta-catenin or APC. Science 1997;275:1787-1790. [PubMed: 9065402]

32. El-Bahrawy M, Poulsom R, Rowan AJ, Tomlinson IT, Alison MR. Characterization of the E-cadherin/ catenin complex in colorectal carcinoma cell lines. Int. J. Exp. Pathol 2004;85:65-74. [PubMed: 15154912]

33. Feig LA. Tools of the trade: use of dominant-inhibitory mutants of Ras-family GTPases. Nat. Cell Biol 1999;1:E25-E27. [PubMed: 10559887]

34. Sjoblom T, et al. The consensus coding sequences of human breast and colorectal cancers. Science 2006;314:268-274. [PubMed: 16959974]

35. Etienne-Manneville S, Hall A. Rho GTPases in cell biology. Nature 2002;420:629-635. [PubMed: 12478284]

36. Knudson AG. Two genetic hits (more or less) to cancer. Nat. Rev. Cancer 2001;1:157-162. [PubMed: 11905807]

37. Doublie S. Preparation of selenomethionyl proteins for phase determination. Methods Enzymol 1997;276:523-530. [PubMed: 9048379]

38. Snyder JT, Singer AU, Wing MR, Harden TK, Sondek J. The pleckstrin homology domain of phospholipase C-beta2 as an effector site for Rac. J. Biol. Chem 2003;278:21099-21104. [PubMed: 12657629]

39. Otwinowski Z, Minor W. Processing of x-ray diffraction data collected in oscillation mode. Methods Enzymol 1997;276:307-326.

40. Sheldrick, GM. SHELXS86-Program for Crystal Structural Solution. Germany: University of Gottingen; 1986.

41. Collaborative Computational Project, Number 4. The CCP4 suite: programs for protein crystallography. Acta Crystallogr. D Biol. Crystallogr 1994;50:760-763. [PubMed: 15299374]

42. Cowtan KD. DM: an automated procedure for phase improvement by density modification. Joint CCP4 ESF-EACBM Newsl. Protein Crystallogr 1994;31:34-38.

43. McCoy AJ, Grosse-Kunstleve RW, Storoni LC, Read RJ. Likelihood-enhanced fast translation functions. Acta Crystallogr. D Biol. Crystallogr 2005;61:458-464. [PubMed: 15805601]

44. Emsley P, Cowtan K. Coot: model-building tools for molecular graphics. Acta Crystallogr. D Biol. Crystallogr 2004;60:2126-2132. [PubMed: 15572765]

45. Murshudov GN, Vagin AA, Dodson EJ. Refinement of macromolecular structures by the maximumlikelihood method. Acta Crystallogr. D Biol. Crystallogr 1997;53:240-255. [PubMed: 15299926] 
a

$12^{1}$

ASEF 408 LQKMIDISLDGFLITPVQKICKYP

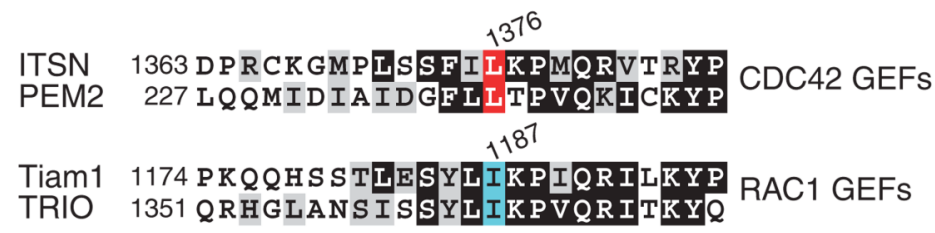

b
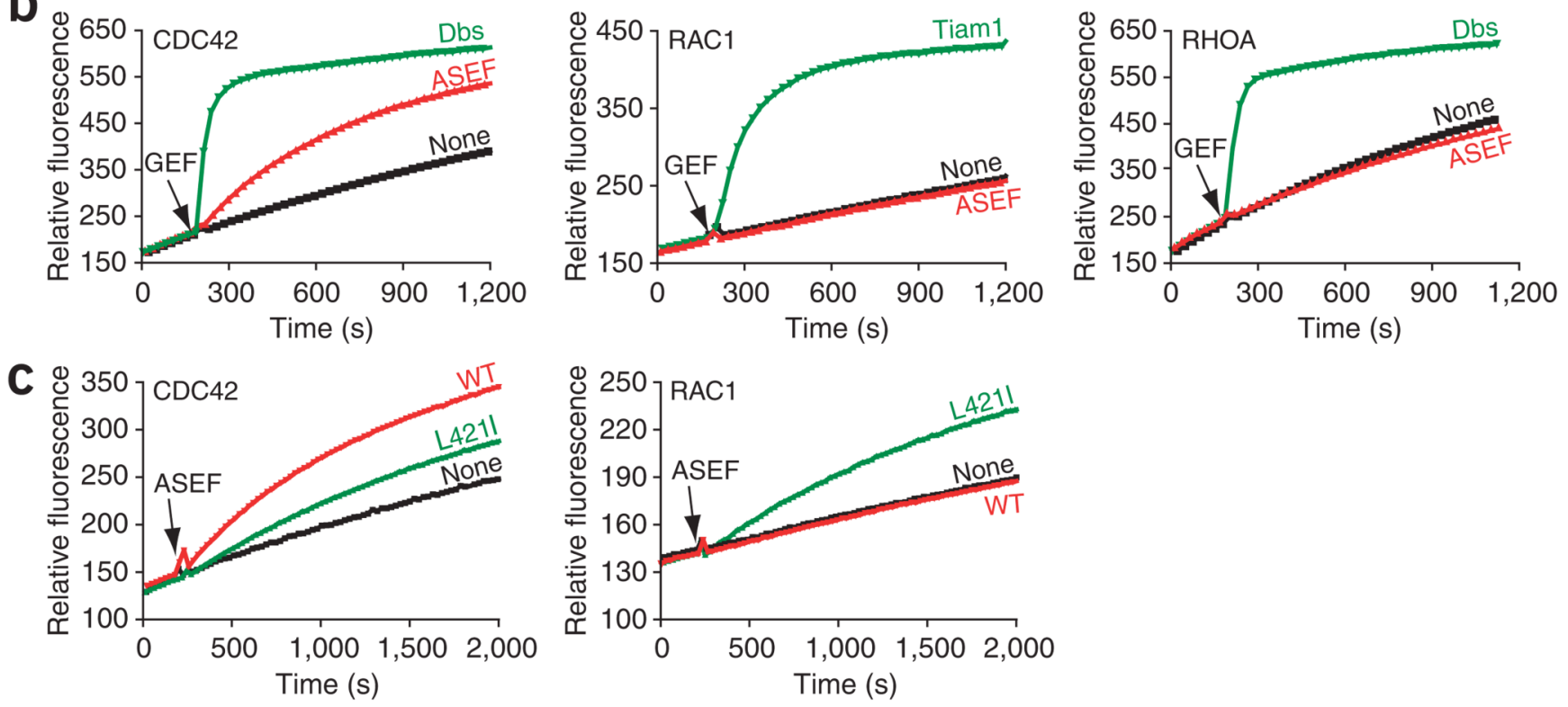

Figure 1. ASEF is a GEF specific for $\mathrm{CDC42}$

(a) Primary sequence analysis suggests that ASEF is specific for CDC42. Shown is sequence alignment of specificity-determining region within select DH domains. GEFs specific for CDC42 have a leucine (red), whereas RAC1-specific GEFs have an isoleucine (blue), in the position previously shown to be important for RAC1 selectivity in Tiam1 (Ile1187). (b) ASEF specifically catalyzes nucleotide exchange on CDC42 but not RAC1 or RHOA. The isolated DH and PH domains of ASEF were used in fluorescence-based GEF assays that monitor the incorporation of mant-GTP into recombinant Rho GTPases. The DH/PH domains of Dbs and Tiam1 were used as positive controls. Arrow indicates time of GEF addition. (c) L421I mutation in ASEF confers the capacity to activate RAC1 at the expense of CDC42 activation. WT, wild-type. 


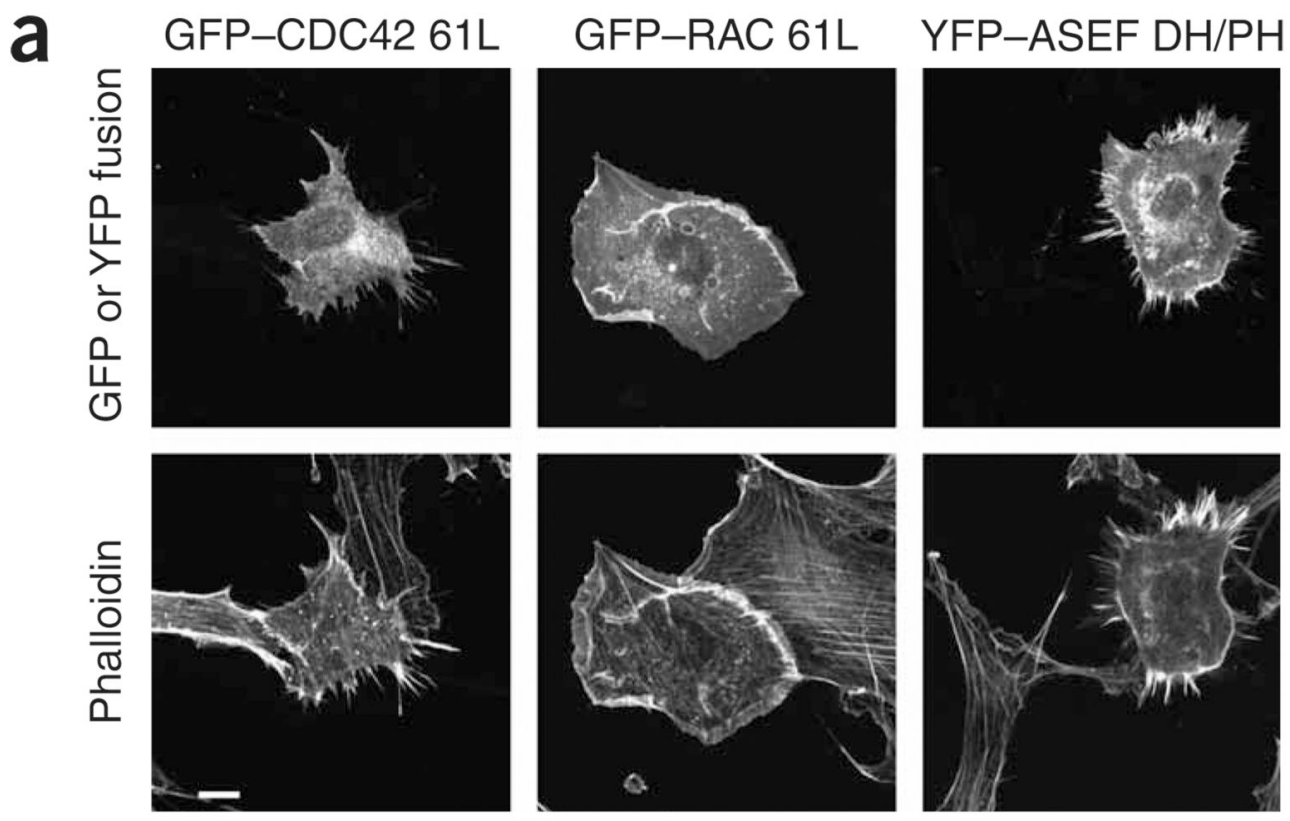

b

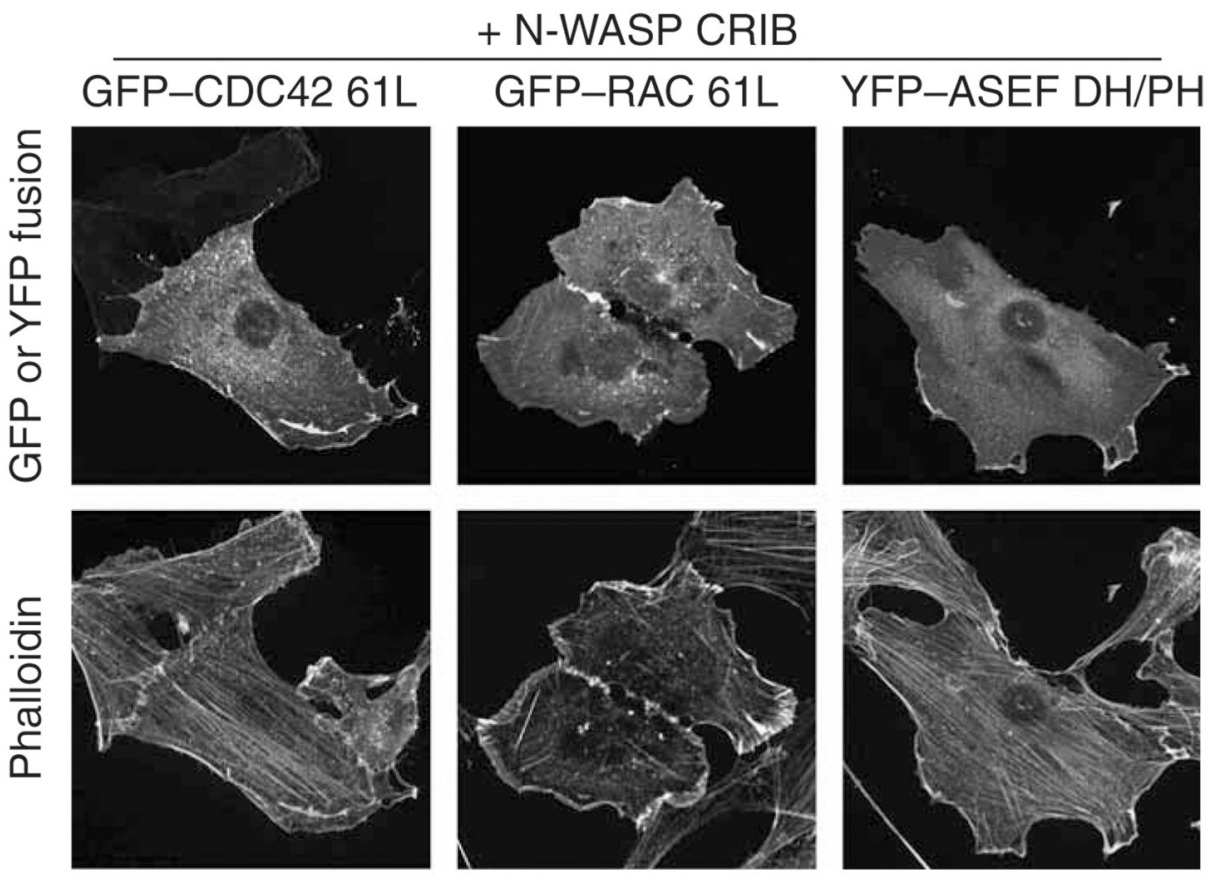

Figure 2. ASEF induces filopodia in NIH3T3 fibroblasts

(a) NIH3T3 fibroblasts were transfected with vectors expressing GFP-tagged, constitutively active CDC42 or RAC1, or YFP-tagged ASEF DH/PH, and stained with Alexa 594-conjugated phalloidin to visualize the actin cytoskeleton. (b) Coexpression of the CRIB domain of the CDC42-specific effector protein N-WASP blocked filopodia formation induced by activated CDC42 and ASEF. Scale bar, $10 \mu \mathrm{m}$. 


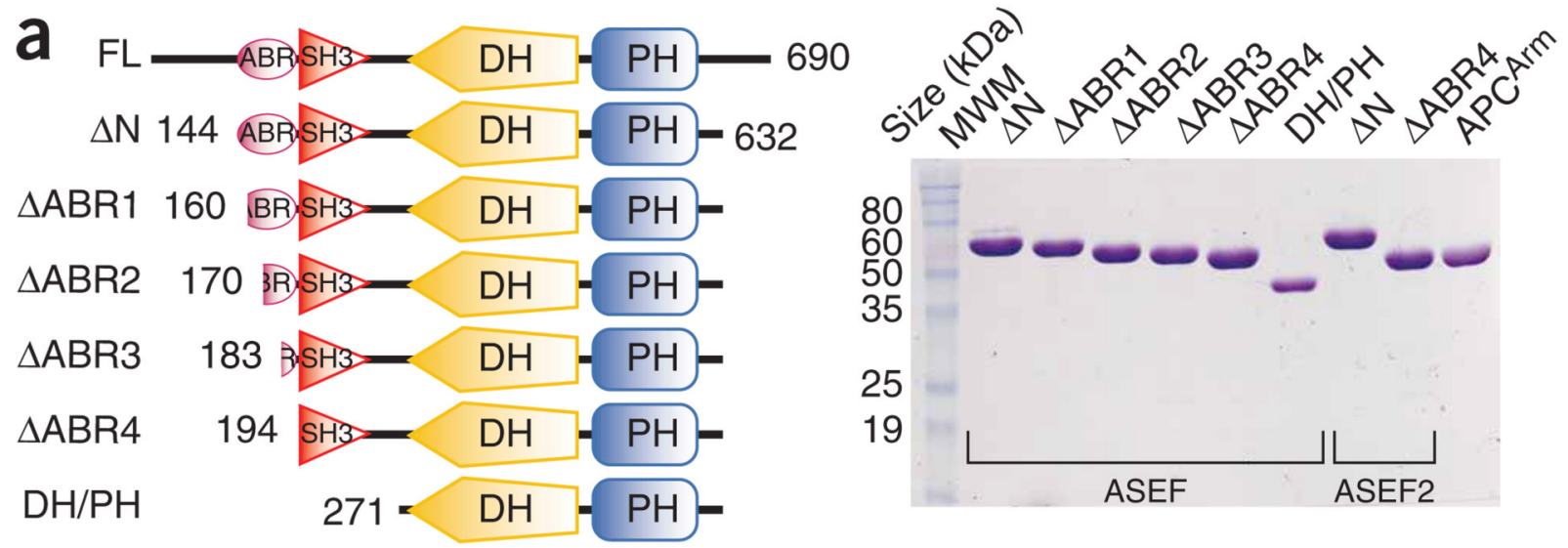

b
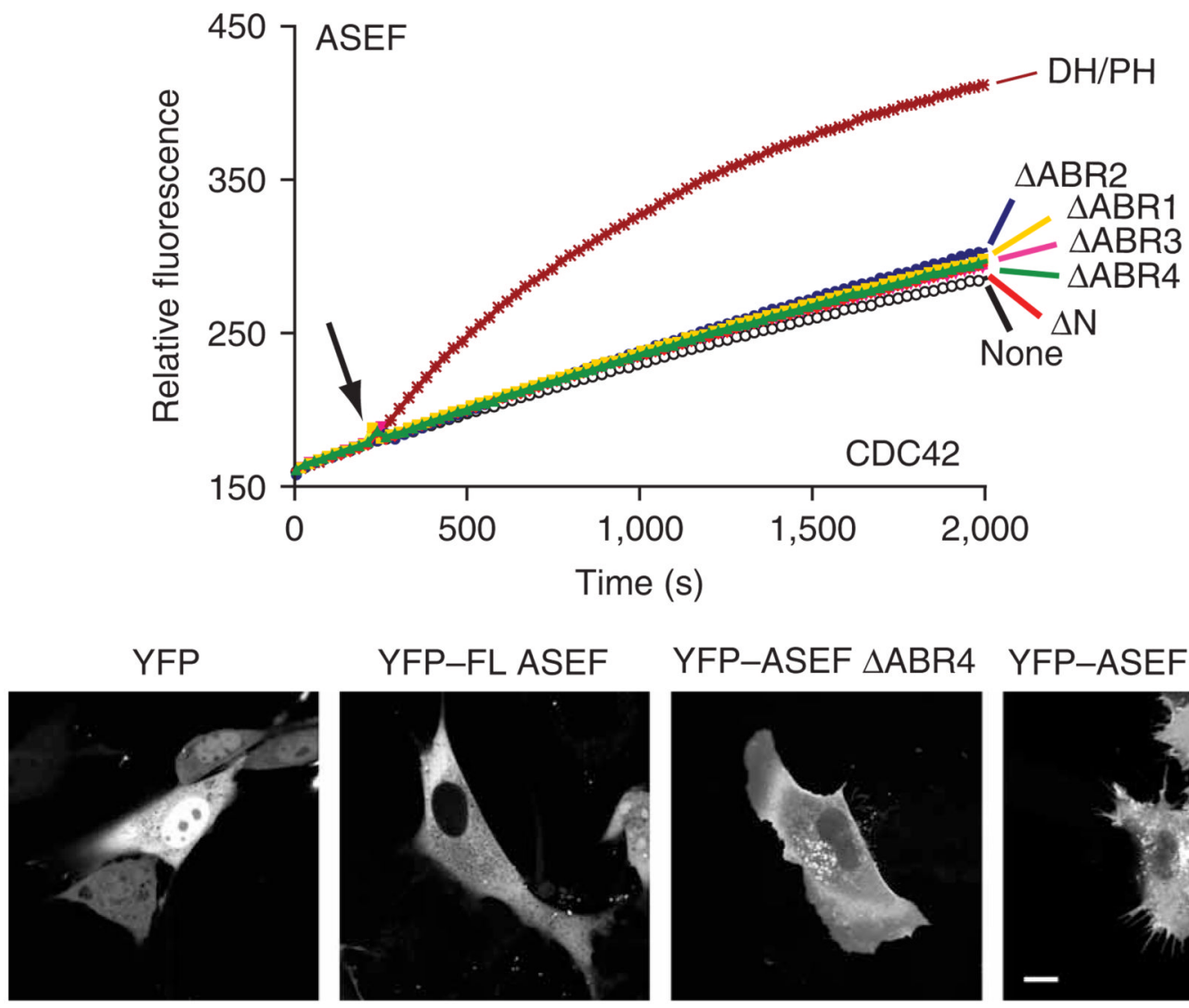

$$
\text { YFP-FL ASEF }
$$
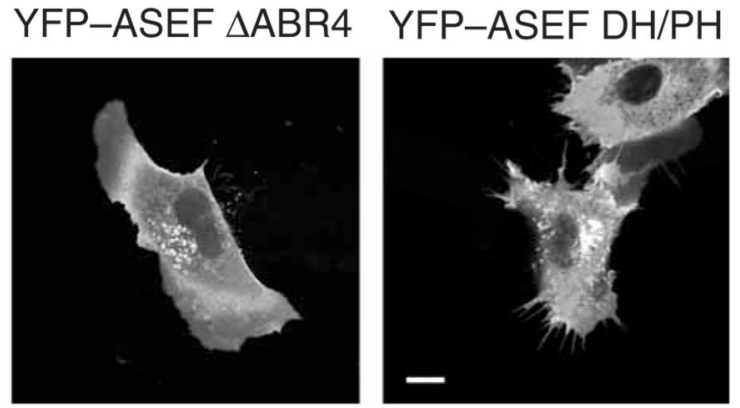

Figure 3. ASEF is autoinhibited by the SH3 domain

(a) Left, schematic representations of N-terminal truncation mutants of ASEF used in this study. Position numbers for ASEF correspond to those published for ARHGEF4 (ref. ${ }^{17}$ ). Right, $5-\mu \mathrm{g}$ samples of recombinant, bacterially produced ASEF, ASEF2 (residues 92-587, $\Delta \mathrm{N} ; 147-$ 587, $\triangle \mathrm{ABR} 4$ ) and APC ${ }^{\mathrm{Arm}}$ (residues 309-798) proteins used in this study were separated by SDS-PAGE and visualized by staining with Coomassie blue to confirm their purity. MWM, molecular weight marker. (b) The GEF activity of N-terminal truncation mutants of ASEF toward CDC42, determined by fluorescence-based GEF assays as in Figure 1b. (c) Deletion of the SH3 domain is required for ASEF activation. NIH3T3 fibroblasts were transiently 
transfected with constructs expressing YFP alone or YFP-tagged full-length and N-terminally truncated ASEF mutants and imaged live to visualize filopodia formation. Scale bar, $10 \mu \mathrm{m}$. 


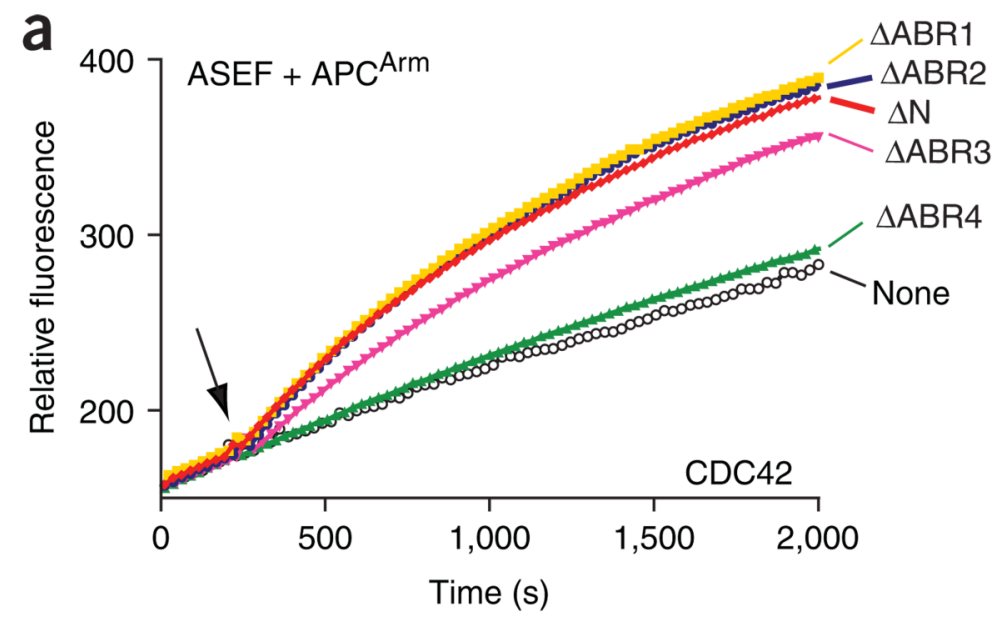

b
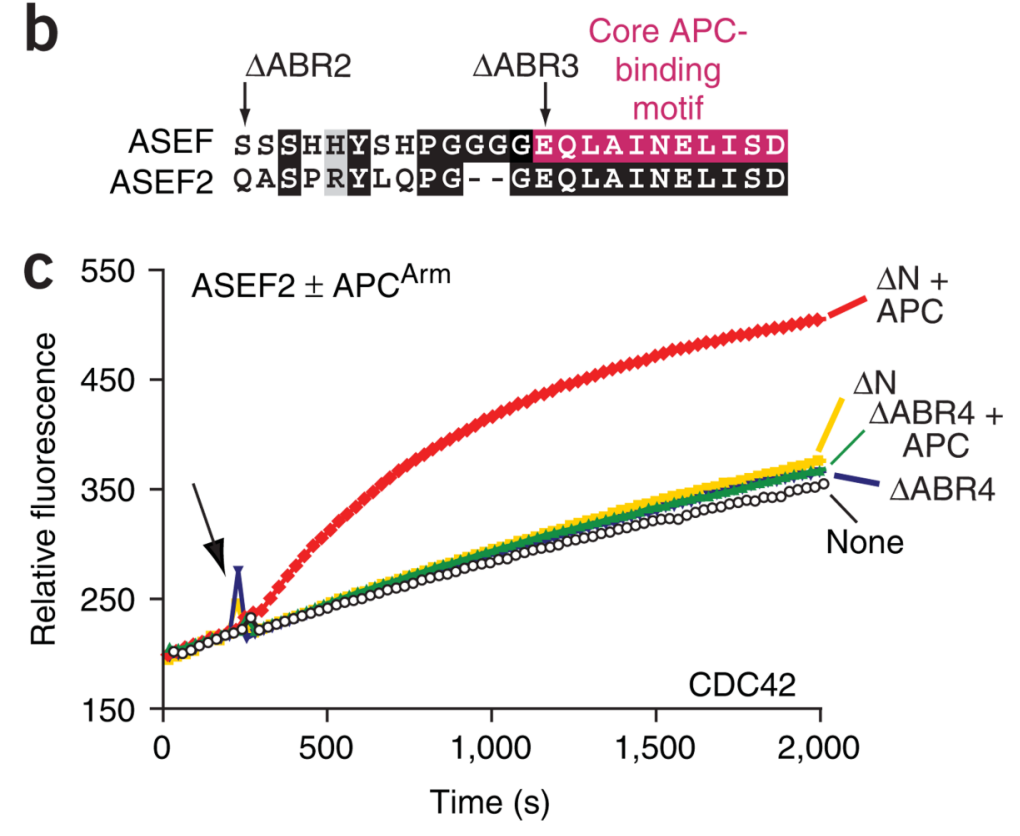

Figure 4. Identification of CAP motif in ASEF and ASEF2

(a) Stimulation of ASEF truncation mutants by APC Arm. The GEF activity of ASEF stimulated by APC was determined using fluorescence-based GEF assays as in Figure 1b. (b) Sequence alignment of the CAB motifs from ASEF and ASEF2. (c) Exchange activity of ASEF2 proteins toward CDC42 in the presence and absence of APC ${ }^{\mathrm{Arm}}$. 
a

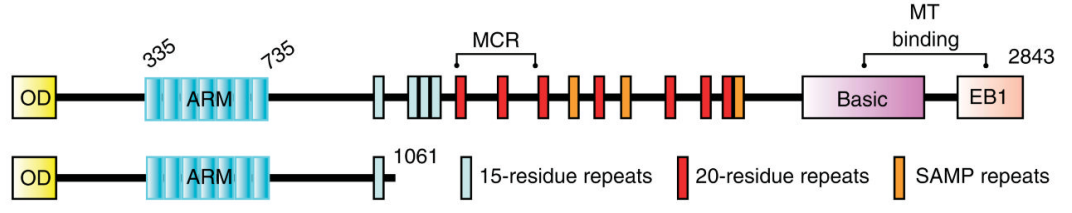

b
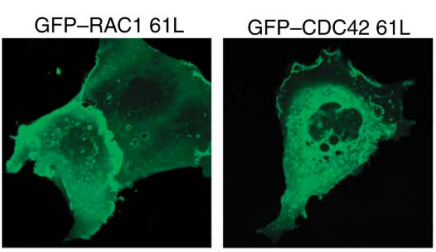

C

mCherry
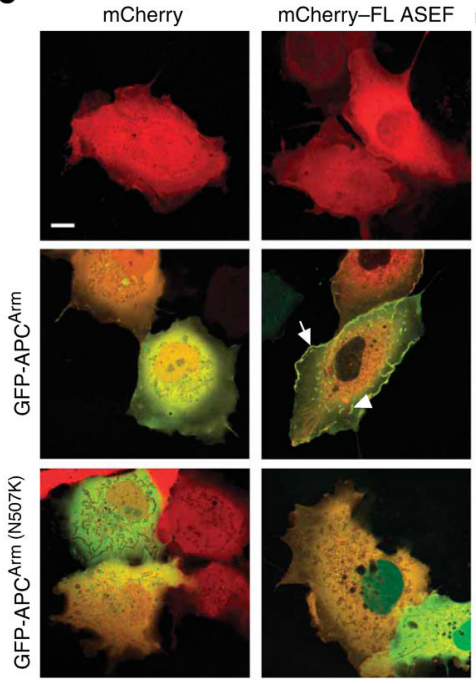
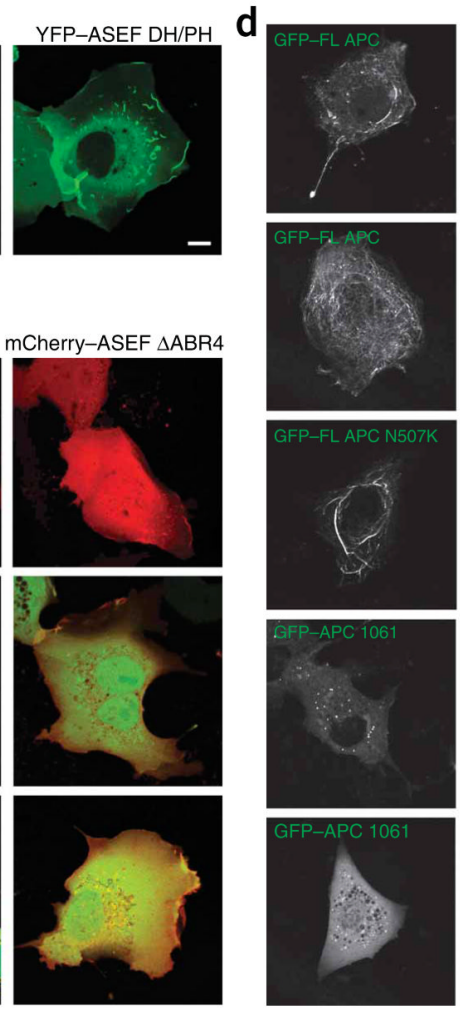
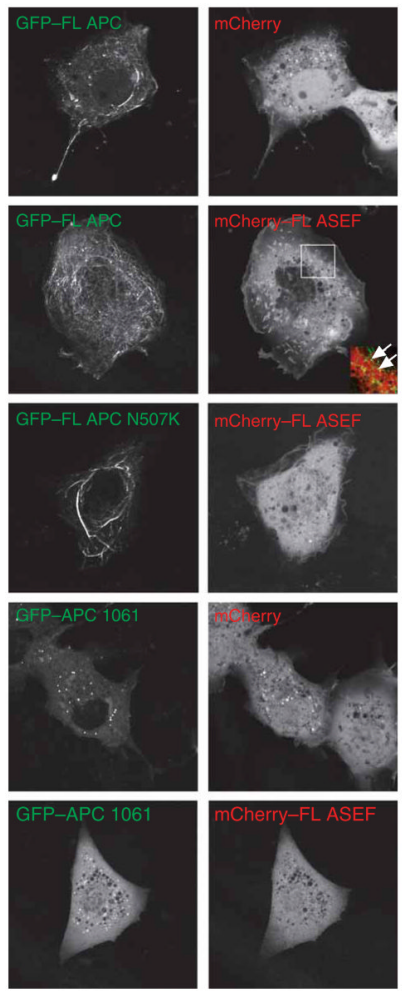

Figure 5. Differential activation of ASEF by full-length and truncated APC

(a) Schematic representations of full-length and truncated human APC. Top, APC contains oligomerization domain (OD), armadillo-repeat domain (ARM), regions for $\beta$-catenin binding (15-residue repeats) and downregulation (20-residue repeats), sequences for Axin binding (SAMP repeats), basic domain that interacts directly with microtubules (MT), and EB1-binding region for indirect association with MTs. Bottom, APC is often mutated within the mutational cluster region (MCR) in cancers, leading to truncated protein. APC 1061 is a truncated form of APC that results from a common germline mutation. (b) Activated ASEF and CDC42 induce similar ruffles in COS-7 cells. COS-7 cells expressing constitutively active RAC1, CDC42 or ASEF $\triangle \mathrm{SH} 3$ were imaged live. In each case, the bottom $z$-section $(0.4 \mu \mathrm{m}$ thick) is shown. Scale bar, $10 \mu \mathrm{m}$. (c) Full-length ASEF is activated by the ARM domain of APC. COS-7 cells were cotransfected with vectors expressing mCherry alone or mCherry-ASEF (FL or SH3containing $\triangle \mathrm{ABR} 4$ ) and GFP-APC ${ }^{\text {Arm }}$ (wild-type or ASEF binding-deficient variant N507K) and imaged live. Arrows indicate that wild-type APC ${ }^{\mathrm{Arm}}$ is recruited to the plasma membrane together with FL ASEF. Scale bar, $10 \mu \mathrm{m}$. (d) Full-length, but not truncated, APC colocalizes with ASEF to produce membrane ruffles. COS-7 cells were cotransfected with vectors expressing mCherry alone or mCherry-FL ASEF and GFP-FL APC (wild-type or N507K mutant) or GFP-APC 1061 and imaged live. Color inset shows that full-length APC and ASEF colocalize to puncta that have been previously characterized as tips of microtubules. 
a
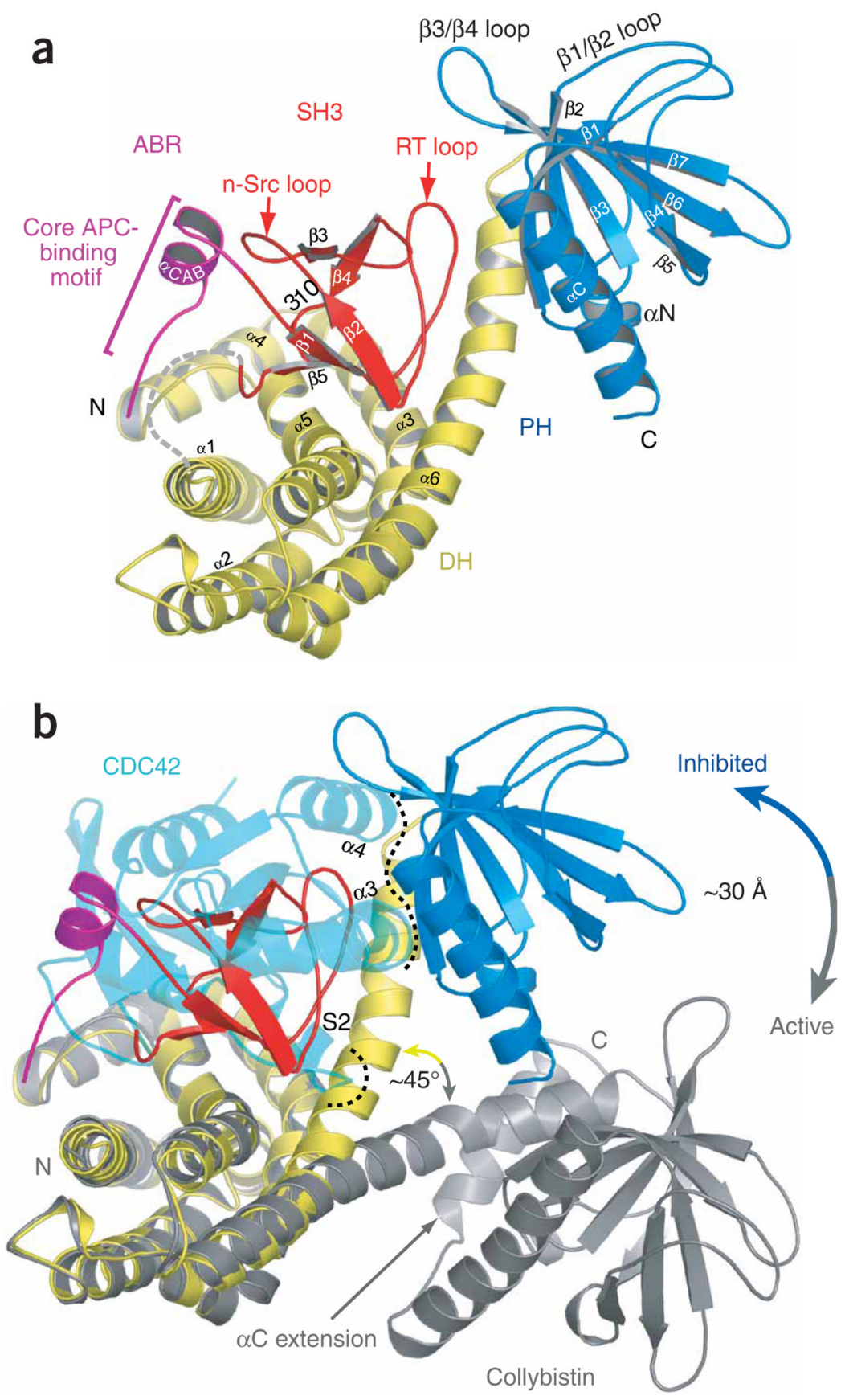

Figure 6. Structure of autoinhibited ASEF

(a) Ribbon diagram of ASEF. The SH3 domain forms an extensive interface with the DH and PH domains. The core APC-binding motif within the ABR contains a small $\alpha$-helix ( $\alpha \mathrm{CAB}$ ) that rests against the $\mathrm{n}-\mathrm{Src}$ loop of the $\mathrm{SH} 3$ domain. Dashed line represents disordered linker region between the SH3 and DH domains. (b) Comparison of autoinhibited ASEF with collybistin bound to CDC42. The structure of the collybistin DH and PH domains bound to CDC42 (PDB 2DFK) was superimposed on the structure of ASEF by aligning their DH domains. In this conformation, the DH and PH domains of ASEF could not bind CDC42 owing to steric clash, and large conformational differences are evident between the inhibited (ASEF) 
and activated (collybistin) states. Dashed lines indicate sites of steric clash; S2 is switch 2 in CDC42. 
a

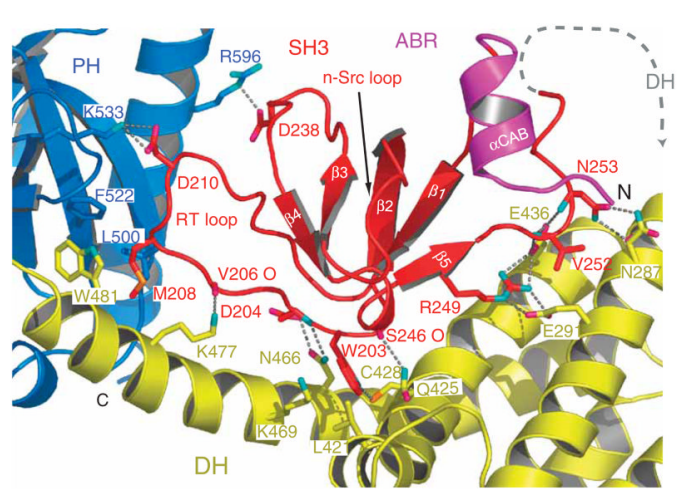

b
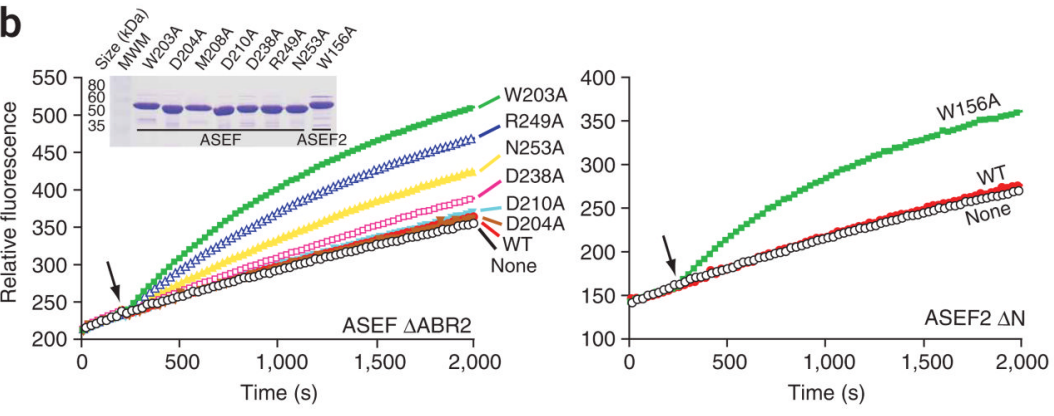

C
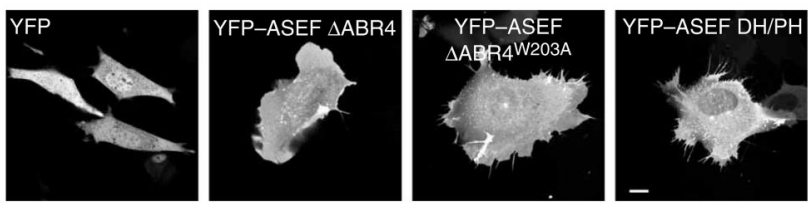

Figure 7. Detailed view of the SH3 domain interface

(a) Molecular interactions between the SH3 domain and DH and PH domains of ASEF. Dashed lines indicate hydrophilic interactions ( $\leq 3.2 \AA$ except for Trp203, which interacts at $3.4 \AA$ ).

(b) Functional analysis of residues within the SH3 domain interface. Substitutions in the SH3 domain of ASEF $\triangle \mathrm{ABR} 2$ were made to selectively disrupt the inhibitory interface and activate the GEF activity of ASEF for CDC42 in the absence of APC (inset shows purified mutants on Coomassie-stained gel; MWM, molecular weight markers). Trp156 in ASEF2 is analogous to Trp203 in ASEF, and W156A in ASEF2 $\triangle \mathrm{N}$ similarly activates the GEF activity of ASEF2. For comparison, exchange reactions in the presence of wild-type GEF (WT) and no GEF (none) are shown. Arrows point to time of GEF addition to the reactions. (c) ASEF W203A activates CDC42 in vivo. Cells were imaged live using fluorescent confocal microscopy. Scale bar, 10 $\mu \mathrm{m}$. 
a

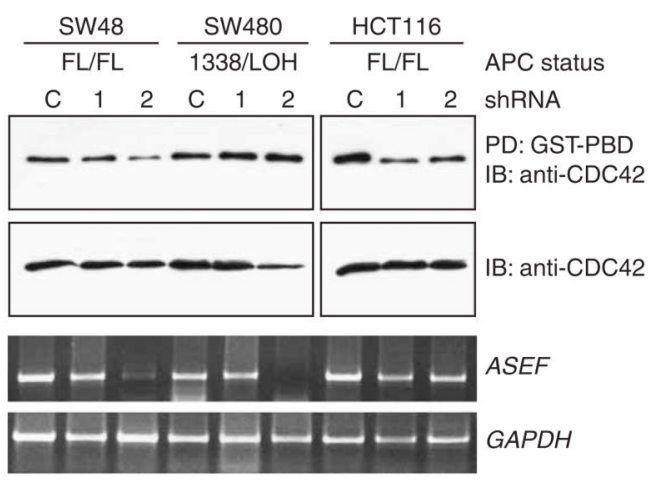

b

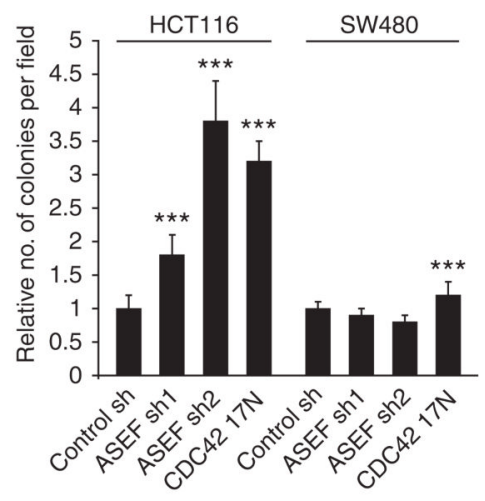

C

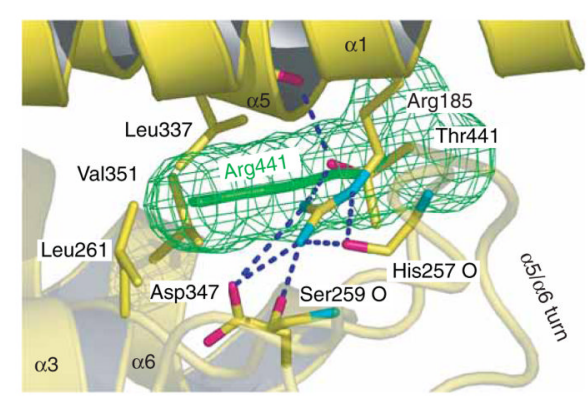

d

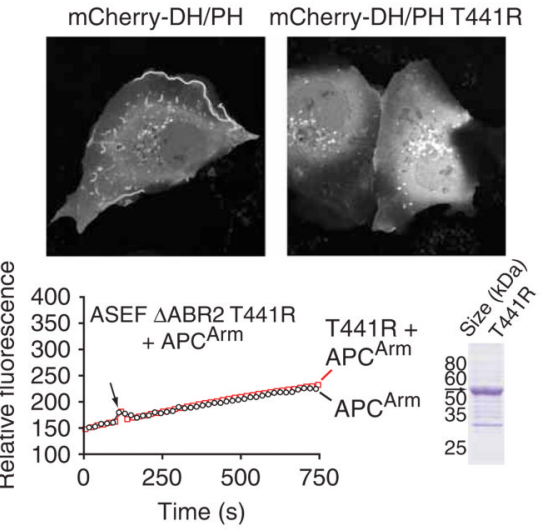

Figure 8. ASEF is a candidate tumor suppressor

(a) ASEF is essential for CDC42 activation in CRC cells containing full-length APC but not those with truncated APC. shRNA was used to knock down ASEF expression in CRC cell lines expressing full-length (SW48 and HCT116) or truncated APC (SW480). CDC42-GTP abundance was assessed by affinity pull-down assays (see Methods) in cells stably expressing shRNA-1, shRNA-2 or shRNA-C (control; see text). ASEF knockdown was confirmed by reverse-transcription PCR (GAPDH was used as a control; bottom gels). (b) ASEF knockdown promotes anchorage-independent growth of HCT116 but not SW480 cells. Cells stably expressing control or $A S E F$-specific shRNAs were assayed for their ability to grow in soft agar. The HA-tagged dominant-negative mutant HA-CDC42 17N was used as a control. Data from three independent experiments are presented as the mean number of colonies per field normalized to the number in an experiment with control shRNA. Error bars represent s.e.m. $* * * P<0.001$. (c) Structural representation of ASEF T441R, a tumor-associated mutant. Thr441 participates in a network of hydrogen bonds that stabilize one end of the DH domain (yellow) of ASEF. When Thr441 is mutated to arginine, this network, as well as hydrophobic core interactions, are disrupted, and this probably results in the unfolding of the DH domain. Arg441 was modeled in silico and is shown as sticks with green mesh van der Waals surface. (d) The T441R mutation inactivates ASEF in vivo and in vitro. Top, COS-7 cells were transfected with a constitutively active ASEF (mCherry-DH/PH) or T441R mutant and analyzed as in Figure 5b. Bottom, The T441R mutation inactivates ASEF in vitro. $\triangle \mathrm{ABR} 2$ T441R was inactive in fluorescence-based GEF assays when stimulated with APC ${ }^{A r m}$, 
performed as in Figure 3b. Graph shows results of assays; Coomassie-stained gel shows purity of mutant construct. 
Table 1

Data collection, phasing and refinement statistics

\begin{tabular}{|c|c|c|c|c|}
\hline & Native & & SeMet & \\
\hline \multicolumn{5}{|l|}{ Data collection } \\
\hline Space group & $C 2$ & & $C 2$ & \\
\hline \multicolumn{5}{|l|}{ Cell dimensions } \\
\hline$a, b, c(\AA)$ & $100.4,79.9,67.4$ & & $116.4,80.5,101.5$ & \\
\hline \multirow[t]{2}{*}{$\alpha, \beta, \gamma\left(^{\circ}\right)$} & $90.0,123.0,90.0$ & & $90.0,104.5,90.0$ & \\
\hline & & Peak & Inflection & Remote \\
\hline Wavelength & 1.5418 & 0.97925 & 0.97936 & 0.97164 \\
\hline Resolution $(\AA)$ & $58-2.25(2.34-2.25)$ & $50-2.20(2.28-2.20)$ & $50-2.20(2.28-2.20)$ & $50-2.20(2.28-2.20)$ \\
\hline$R_{\text {sym }}$ & $4.1(41.2)$ & $7.7(29.8)$ & $7.6(38.1)$ & $6.8(47.1)$ \\
\hline$I / \sigma I$ & $30.2(3.2)$ & $26.7(4.9)$ & $25.7(3.7)$ & $24.4(2.7)$ \\
\hline Completeness (\%) & $99.1(97.9)$ & $99.6(97.4)$ & $99.0(93.5)$ & $98.0(86.9)$ \\
\hline Redundancy & 2.8 & 7.2 & 7.0 & 6.8 \\
\hline \multicolumn{5}{|l|}{ Refinement } \\
\hline Resolution ( $\AA$ ) & $15.0-2.25$ & & & \\
\hline No. reflections & 19,854 & & & \\
\hline$R_{\text {work }} / R_{\text {free }}$ & $21.7 / 27.9$ & & & \\
\hline \multicolumn{5}{|l|}{ No. atoms } \\
\hline Protein & 3,330 & & & \\
\hline Water & 100 & & & \\
\hline \multicolumn{5}{|l|}{$B$-factors } \\
\hline Protein & 51.3 & & & \\
\hline Water & 48.2 & & & \\
\hline \multicolumn{5}{|l|}{ R.m.s. deviations } \\
\hline Bond lengths $(\AA)$ & 0.008 & & & \\
\hline Bond angles $\left({ }^{\circ}\right)$ & 1.063 & & & \\
\hline
\end{tabular}

X-ray diffraction data were collected from two crystals. Values in parentheses are for the highest-resolution shell. 